\title{
Predicting Suitable careers through use of Snakes and Ladder Game
}

\author{
P. Vamsi ${ }^{1}$, Rohit Desai ${ }^{2}$, G Sai Sampath ${ }^{3}$, V., J.K.R. Sastry ${ }^{4}$, Chandra Prakash ${ }^{5}$ \\ KoneruLakshmaiah Education Foundation, Vaddeswaram, AP, India, vamsipuppala4599@gmail.com \\ KoneruLakshmaiah Education Foundation, Vaddeswaram, AP, India, rohitdesai1999@ gmail.com \\ KoneruLakshmaiah Education Foundation, Vaddeswaram, AP, India, sampathgurujala@ gmail.com \\ KoneruLakshmaiah Education Foundation, Vaddeswaram, AP, India, drsastry@kluniversity.in \\ KoneruLakshmaiah Education Foundation, Vaddeswaram, AP, India, vchandrap@kluniversity.in
}

\begin{abstract}
Choice of a career in the Initial stages of the graduating a professional degree plays long lasting impact on a student and has great impact on the very life of the student. Predicting suitable careers to the students involves assessment of Psychology of the studentsas every job requires some level of psychology so that the student would be able to carry on the Job to the utmost satisfaction of the employers.
\end{abstract}

Games are quite frequently used for assessing the Psychology of the students. Snakes and ladder Games played by the students is primarily dependent on Luck. The game playing is modified through addition of rules such that the Game is played based on the knowledge and intelligence of the student. A cognitive model for assessment of Psychology of the student based on modified Snakes and ladder Game and an expert system is presented that combines different Psychological factors and provides a platform for prediction of suitable careers to the student.

Experiments have been conducted by making several students play the game leading to the prediction of careers suitable to the students. The Prediction accuracy has been found to be $95 \%$

Key words: Snakes and Ladder, expert system, assessing knowledge, evaluating psychological factors, solving puzzles, career prediction, cognitive model, logical thinking, patience and perseverance, learning ability

\section{INTRODUCTION}

In the Contemporary world, Knowledge is the most powerful Weapon which can be achieved only by Learning. As Most of the People get bored by learning new things in the old fashioned manner, we can make them more engaged in the learning Process by adding some fun activity in it. In this way, we can make them learn new things by providing some entertainment through which they can't get bored. Through this fun activity, we can also assess the person's in-depth knowledge in a subject and his/her Psychological factors and can predict the suitable Career for him/her.

Games are artificial Intelligence constrained based models that help the students gain ability to solve the problems through use of search methods focussed at achieving some target set to achieve. The characteristics/cognitive aspects of a person playing the Games can also be measured using the games.

Snakes and Ladders are an ancient Indian board game regarded today as a worldwide classic. It is played between two or more players on a gameboard having numbered, gridded squares. A number of "ladders" and "snakes" are pictured on the board, each connecting two specific board squares. The picture of the Game playing surface is shown in Figure-1.

A die is used by the Players to decide on the Number of steps to move. If the movement leads to a square where a ladder exits, the player is moved to the Top of the Ladder Square and if the movement leads to head of a snake the player is move to the square where the tail of the Snakes exists. The Game is played till a player reaches Last Square.

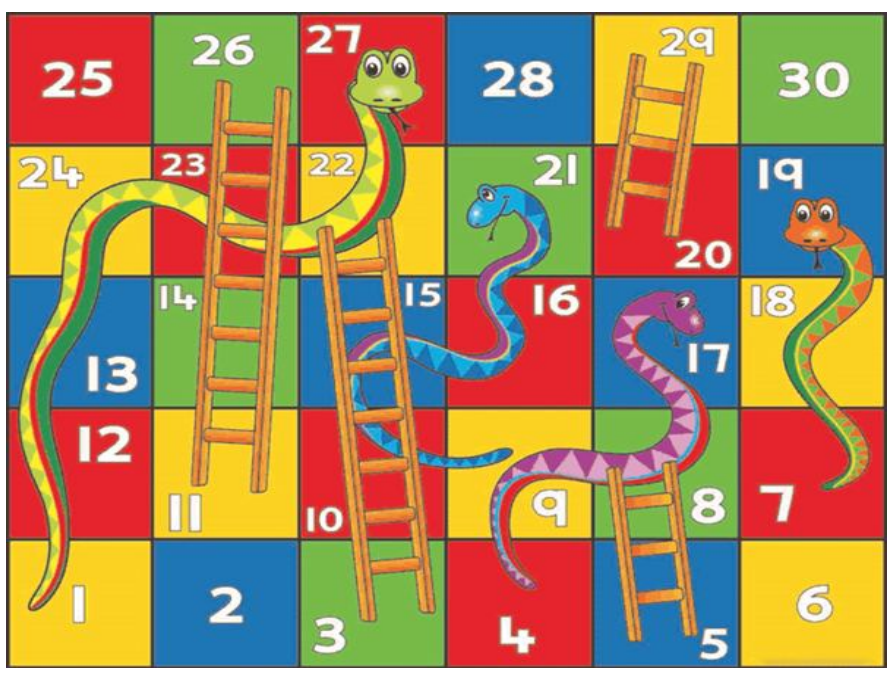

Figure 1: Picture of Snakes and Ladder Game 
P. Vamsi et al., International Journal of Emerging Trends in Engineering Research, 8(5), May 2020, 2065 - 2073

Psychological evaluation is a method to assess an individual's behaviour, personality, cognitive abilities, and several other domains. A common reason for a psychological evaluation is to identify psychological factors that may be inhibiting a person's ability to think, behave, or regulate emotion functionally or constructively. It is the mental equivalent of physical examination. Other psychological evaluations seek to better understand the individual's unique characteristics or personality to predict things like workplace performance or customer relationship management. Evaluating Psychological factors play a crucial role in students' life for career prediction.

It is pointed out that evaluating psychological factors using games or puzzles are the best way. Psychological assessment of a student's requires capturing data such as the number of times the game is played, number of times the game is won, number of questioned answered correctly and wrongly, total time taken for playing the game etc. while the game is played and a cognitive model is used for assessing the Psychological level of the students using the data collected while the Game is played.

In this paper, the authors proposed that a game called 'Snakes and Ladder' is best to assess the Knowledge, Learning Ability,Patience,Competitive Spirit etc. of a student and also pointed out that evaluating students in all different aspects is useful to recommend/decide the right job which suits them.

An expert system is required that combines the Psychological level of different cognitive aspects of the students such that each mapping can be mapped to a suitable career. Exceptions contained in the mapping needs to be eliminated.Job prediction involves finding different careers and mapping of the Job to aa combination of Levels of different PsychologicalFactors.

\section{PROBLEM DEFINITION}

The main problem is to determine the computations that needs to be done while the snakes and Ladder Game is played using the modified rules and also building a cognitive model that can be used to assess different cognitive levels of the students based on the computations made and laso combining the psychological levels of different factors such that a combination is mapped to a suitable career so that the career that is suitable to student can be predicted.

\section{RELATED WORK}

Selecting a proper career is very important for a student for a proper life settlement. Generally, a human expert in that field suggests a career options to the students based upon their academic standards. But a human expert can also make errors in suggesting the most suitable career to the students. The expert system can build using AI techniques can be used to replace the human expert.
AI based expert system can suggest best suitable career to a student through construction of a cognitive model suiting to a student. That model can be generated through assessment of various psychological factors viz. logical thinking, learning ability, decision making, patience and perseverance, etc. solving puzzles is one of the most suitable methods to assess them. The following provides an examination on this direction.

In AI, the expert system uses the knowledge domain stored in the computer in order to make a meaningful decision for the provided problem. It is a computer system that uses human knowledge and replaces the human expert. Application of expert system is in wide range viz. education, agriculture, medical, industry, etc.

Balasubramanyam, S., Padmaja Usharani, D., et al. [1] pointed that the expert system resembles human expert knowledge.Aaron T Beck, Emily AP Haigh [2] declared that the behavioural model of Beck was used to formulate and treat psychological disorders. The universal behavioural model is a collection of common concepts that can be useful across the mental illness continuum. Lent, Robert W, Ijeoma Ezeofor et al.[3] stated two studies on the social cognitive model of self-management careers (Brown \& Lent, 2013) were presented for a career prediction and decision making factors in the college students.

Steven K. Mtsweni and Johnson O. Dehinbo [4] pointed out that selecting the best suitable career(s) by the student is the crucial step that must be taken care of while opting for them. But a major mistake made by the student is following their known seniors for selecting their career(s), which may not suit most of them because of various factors. Therefore, an expert system needs to be developed that can evaluate a student's ability and direct him/her in choosing the most suitable career.

Chathra Hendahewa, MaheshikaDissanayake et al.[5] created an Expert System of Career Advisory Program, called iAdvice, to direct students in their higher education in order to decide their career paths and choose their subjects in line with their career objectives. The expert system features such as alternative solutions with explanations, reasoning ability, measurement of probability and providing uncertainty, and the ability to question is found in iAdvice

Manisha $\mathrm{L}$ et al., [6] have presented a theoretical framework that show the way careers can be selected to the students. El Haji, Essaid et al., [7] have presented a "Multi-expert system design for educational and career guidance which is an approach based on a multi-agent system and ontology.

Saraswathi, S., et al., [8] have presented Design of an online expert system for career guidance and Ezenkwu, et al., [9] have presented an Automated Career Guidance Expert System Using Case-Based Reasoning. Athanasios et al., [10] have presented an Expert system for job matching of the unemployed.". 
P. Vamsi et al., International Journal of Emerging Trends in Engineering Research, 8(5), May 2020, 2065 - 2073

Essaid El Haji et al., [11] have presented an Expert system design for educational and vocational guidance, using a multi-agent system. Sripath Roy, et al., [12] have presented a Student career prediction using advanced machine learning techniques. Most of the approaches presented in the literatureare eithernot based on cognitive models or combined with the expert models

Chandra Prakash V et al., [13] have presented on the applicability of Sokoban Game for prediction suitable careers to the students. Chandra Prakash V et al., [14], Dr.SasiBhanuJ et al., [15] have presented models that assess the applicability of Suduko Game for building the cognitive models that can be used for predicting the careers suitable to the students

Chandra Prakash V., J.K.R. Sastry et al. [16] stated that the expert system replicates the behavior of a human expert, which helps to assess different types of psychological factors of persons for a particular purpose of classification. In this paper, they proposed an expert system for career guidance by using 8-puzzle solving. They have pointed that the cognitive model simulates the psychological factors of the students' viz. speed of solving, learning ability, intelligence, etc. The researcher suggested the system for evaluating factors by conducting the game for a student and builds the cognitive model of the student for suggesting a suitable career.

Dr. J. SasiBhanu et al., [17] [18[ have presented methods that can be used for providing Career Guidance through making the students play TIC-TAC-TOE Game, They have constructed a cognitive Models that can be used for Psychological levels of the students which are used for building an expert systems. The expert systems are used for predicting the careers of the students

Chandra Prakash et al., [19] have shown how the memory of a student can be assessed and the way the memory power of the students can be used for predicting the careers that are suitable to the students.

Kantha Rao et al., [20] have presented a method using which the concentration levels of the students can be assessed and the same is used for predicting the suitable careers to the students. They have designed and developed a Concentration game for assessing cognitive levels of the students

Chandra Prakash et al., [21] have developed N-Coin puzzle game and develop a method that can be used for assessing the levels of various psychological factors that help computing the cognitive level of the students. An expert a system is also presented that can be used for predicting suitable careers to the students.

Chandra Prakash et al., [22] presented the way the cognitive levels of the students can be assessed considering the assessment of the students in carrying crypto arithmetic. They have also shown the way the cognitive levels can be used for predicting the careers that are suitable to the students.

Many games have been developed and used to assess different psychological factors of the students. The factors assessed differed a lot. Sometimes the same factor is assessed using different games. Chandra Prakash et al., [23] development of a Comprehensive and Integrated Expert System for Career Assessment based on different Cognitive models built through different games

One of the most important issues is to manage the variability of the assessment of the Psychological factors that are computed through different games. The variability needs to be neutralised. The variability can be smoothened through assessing the cognitive levels of the students by using as many games as possible. In this paper Snakes and Ladder game is used for assessing the Cognitive level of the students considering Knowledge, Patience, Learning ability, perseverance, and memorising

\section{INVESTIGATIONS AND FINDINGS}

\subsection{Use of Data Repositories}

The Game is developed using three databases for maintaining Questions with their respective Answers relating to specific Subjects. The Second Database(D2) maintains the number of Questions the user already answered either Right/Wrong, and the Third Database(D3) maintains the list of all the Questions which the User Answered Incorrectly.

The Questions are of two types, Numeric and Non-Numeric. Numeric Questions are to be answered by simply typing on the textbox provided to the User. For example, a Numeric question from Digital Logic Circuit Subject can be displayed as "A person crosses a $600 \mathrm{~m}$ long street in 5 minutes. What is his speed in km per hour?" and the user will provide a numeric answer.

Non-Numeric Questions Requires that the User choose one among several options(MCQ type) displayed to him/her.For Example Non-numeric Question from Data structures \& Algorithm Subject can be displayed as."'The format identifier '\%i' is also used for ___ data type is a) charb) intc) floatd) double" and the user will provide one of the options that they feel correct as the answer

\subsection{Game Playing Style}

The User will be made to play the Game till he/she Wins 10 games. The Questions to be displayed follows a particular Pattern(70-30 Strategy).Of All the Questions to be displayed,70\% of the Questions are taken from the First Database such that they were not answered by the User till then(i.e, It is not included in Third Database). The Remaining 30\% questions are taken from the Third Database which the User Answered Incorrectly.This was made to ensure how much he/she can memorizethe Acknowledged Questions which he/she answered incorrect. 
P. Vamsi et al., International Journal of Emerging Trends in Engineering Research, 8(5), May 2020, 2065 - 2073

\subsection{Modified Rules for Playing Snakes and Ladder Games}

Modified rules for playing Snakes and Ladder Game

The general rules followed for playing the game is modified so that the game is useful for assessing cognitive level of the students. The rules are modified such that Psychological factors of a student that include knowledge,learning ability, patience, competitive spirit and perseverance of a student can be assessed.

Following are the Modified rules of the Game:

1. There are a series of steps starting from 1 to 30 .

2. Few steps contain either the starting or ending of the ladder, it can also contain either snake head or tail, but it doesn't contain both ladder and snake.

3. It is a single player game played by a student with the computer.

4. Both the player and computer start at 1 step. The one who reaches the $30^{\text {th }}$ step is the winner.

5. The movements of the players are decided by a dice (random generator). In each turn they roll a dice and they move forward accordingly. If the final step of the player in his/her turns ends at the starting point of the ladder or head of the snake, he/she must answer a question correctly in order to climb the ladder or escape from the snake respectively. If he/she fails to give the right answer they end up in not climbing the ladder or being bit by a snake.

Whenever a player (excluding computer) lands on "Ladder" block", a question is flagged and if the question is answered correctly, then the player is allowed to move to a square where the top of the ladder exits or else he player stays at the landed location and does not climb the ladder

Similarly whenever a player(excluding computer) lands at the head of the "Snake " block, a question is raised to the student and if the question is answered correctly he stays at the location where the Head of the snake exists or lese the player is move to a block where the tail of the snakes exists.

On the other hand, the computer needs not to answer any questions. If it is at the starting position it will climb the ladder or at the head of the snake it ends up at the tail of the snake.

6. No player should roll the dice until the current player moves his object.

7. A player who rolls six numbers on dice can get another chance.

8. During the last stage of the game, Anyone can win only if he rolls the dice that exactly make him land on the last block,if he rolls more than required he stays there itself.
9. The above said process repeats for each round. After 10 rounds the psychological factors are assessed and results are displayed.

\subsection{Assessing Knowledge}

Knowledge in a specific domain is the key factor to assess a person for any role in various places and hence it plays a crucial role in overall assessment. So this has to be measured precisely. In this game, the knowledge is measured through the no of answers $(\mathrm{Ra})$ the person is able to give correctly on their first appearance (or) it can also be stated as summation of the no of questions he answered correctly in each game. So the more the correct answers the better his score will be in the end. Now we cannot directly include the number(no of questions vary for person to person) as it is only directly proportional. In order to generalise this to every person it is now divided by the total no of unique questions(Uq). This now will be multiplied with 100 in order to get in percentages, so that we can find the difference easily. Mathematically now we can write this as

$\mathrm{K}=(\mathrm{Ra} / \mathrm{Uq}) * 100$

Where

$\mathrm{K}=$ knowledge possessed

$\mathrm{Ra}=\mathrm{No}$ of right answers of questions in their first appearance.

$\mathrm{Uq}=$ no of unique question

The quantitative score $\mathrm{K}$ is then computed to Qualitative score using Table-1. Table- 1 is arrived at using expert model based on the experience of subject expert. As such the conversion table can be obtained by using data mining techniques applied on to data that is captured and maintained while the game playing is in progress. The Table is one part of the expert models for assessing "Knowledge Factor" both quantitatively and qualitatively.

Table 1: Quantitative- Qualitative Conversion of knowledge score

\begin{tabular}{|c|l|l|}
\hline $\begin{array}{c}\text { Serial } \\
\text { Number }\end{array}$ & $\begin{array}{l}\text { Quantitative } \\
\text { Knowledge Score }\end{array}$ & $\begin{array}{l}\text { Qualitative } \\
\text { Knowledge Score }\end{array}$ \\
\hline 1 & $>=75$ and $<=100$ & Distinction \\
\hline 2 & $>=60$ and $<75$ & First class \\
\hline 3 & $>=50$ and $<60$ & Second class \\
\hline 4 & $>=40$ and $<50$ & Third class \\
\hline 5 & $<40$ & Fail \\
\hline
\end{tabular}

\subsection{Assessing Learning Ability and Memorising}

Memorising and learning power are the Psychological factor that paly great role in choosing a creer. Some Jobs especially 
P. Vamsi et al., International Journal of Emerging Trends in Engineering Research, 8(5), May 2020, 2065 - 2073

the IT Jobs requires huge learning in short span of time and the technologies keep changing regularly. The more the memorising power the better the person can maintain the things on finger tips.

In this game, the player will be acknowledged with the right answer and explanation whenever he fails to answer correctly. As the student plays the game, the learning ability is calculated and the average of it is computed at the end of playing the game roughly 100 times.. Learning ability in each game is calculated as the no of questions he answered correctly using 3rd Database in which questions asked and answers provided are stored.

$\mathrm{n}$

$\mathrm{i}=1$

$$
\text { Learning ability }=\Sigma \mathrm{L}_{\mathrm{i}} / \mathrm{n}
$$

Where

$$
\mathrm{L}_{\mathrm{i}}=\mathrm{r} 3 / \mathrm{q} 3
$$

$\mathrm{L}_{\mathrm{i}}=$ learning ability in each game

$\mathrm{r} 3=$ no of questions he answered correctly from the questions of 3rd Database in eachgame

$\mathrm{q} 3=$ the total no of questions he was asked from the third Database in each game.

$\mathrm{N}=$ total no of games played

Note: if $\mathrm{q} 3$ is 0 then $\mathrm{r} 3$ is also 0 then we will take learning ability of the student as 1

\subsection{Assessing Patience}

This expert system assesses the patience by the number of winning games he played.

Patience $=\mathrm{n}$, where $\mathrm{n}=$ no of winning games played. The score thus computed is converted into a qualitative score using the expert system shown in Table 2

Table 2: Conversion of Quantitative patience score to qualitative score

\begin{tabular}{|l|l|l|}
\hline $\begin{array}{l}\text { SN } \\
\text { O }\end{array}$ & $\begin{array}{l}\text { Patience } \\
\text { Quantitative Score }\end{array}$ & Patience Qualitative Score \\
\hline 1 & $>=0$ and $<=3$ & Not accessed \\
\hline 2 & $>=3$ and $<=5$ & Low \\
\hline 3 & $>=5$ and $<8$ & Medium \\
\hline 4 & $>=8$ & High \\
\hline
\end{tabular}

\subsection{Assessing Competitive Spirit and perseverance}

Competitive spirit and perseverance are great weapons for a person who lacks knowledge because they drive the person to cross their limits and motivates them in their downfall. By this expert system we can calculate the perseverance by the total no of games the user won by the total no of games played.

Perseverance $=\mathrm{n} 1 / \mathrm{n} 2$ where $\mathrm{n} 1=$ no of games user won, $\mathrm{n} 2=$ no of games played. The quantitative score is converted to qualitative score using the expert system shown in Table 3.

Table 3: Converting Perseverance quantitative score to qualitative score

\begin{tabular}{|l|l|l|}
\hline S.no & $\begin{array}{l}\text { Perseverance Quantitative } \\
\text { score }\end{array}$ & Qualitative score \\
\hline 1 & $<=0.2$ & very low \\
\hline 2 & $>0.2-<0.4$ & low \\
\hline 3 & $>=0.4-<=0.6$ & medium \\
\hline 4 & $>=0.6$ & high \\
\hline
\end{tabular}

\subsection{Predicting Suitable Careers}

Selecting suitable Career plays a vital role in every Individual's Life. Most of the Younger generation people get stuck, when it comes to choosing the suitable career among all the options available to them in the present World. Since, Our Expert System is designed to predict the best suitable Career fot the student in IT industry field.

Table 4 shows the mapping of Psychological levels required considering patience, perseverance, learning ability and the knowledge level of the students combined with the Academic scoring achieved in the subjects that include data structures, algorithms and Software engineering. The requirement mapping is done for different kinds of IT Jobs that the students can take up.

Where, Distinction indicates 75\%-100\% score, First Class indicates $65 \%-75 \%$ score, Second Class indicates 50\%-65 $\%$ score

So, based on the Scores gained by the Student in each respective Domain,Our expert system will be able to predict the best Suitable Career for him/her which leads to reach a successful Position in his/her life.

\section{RESULTS AND ANALYSIS}

We have selected a random set of Students from the Computer Science \& Engineering Department.They were made to play the modified Snake and Ladder Game provided by our Expert System. The System successfully predicted the student's Psychological factors by generating the OPA(overall Psychological Assessment) score that he/she gained in the subjects of their respective Specialization. It has also made some predictions about their Suitable Career options in their respective specialization based on this OPA 
P. Vamsi et al., International Journal of Emerging Trends in Engineering Research, 8(5), May 2020, 2065 - 2073

score. The results from the Analysis were shown in the Table 5 and Table 6 respectively.

Table 5: Performance of the students during Campus placement

\begin{tabular}{|c|l|l|c|c|}
\hline S. & \multicolumn{1}{|c|}{ Name } & $\begin{array}{c}\text { Registration } \\
\text { Number } \\
\text { O }\end{array}$ & $\begin{array}{c}\text { S.G.P.A } \\
\text { (Max } \\
\text { Grade } \\
10.0)\end{array}$ & $\begin{array}{c}\text { Annual- } \\
\text { Salary } \\
\text { Package that } \\
\text { he/she Gained }\end{array}$ \\
\hline 1 & K.Sumanth & 170031161 & 9.0 & $6,00,000$ \\
\hline 2 & $\begin{array}{l}\text { Rishi Ramana } \\
\text { Sai }\end{array}$ & 170030118 & 7.6 & $6,40,000$ \\
\hline 3 & C. Akhil & 170030562 & 8.2 & $4,00,000$ \\
\hline 4 & ch Naga Saketh & 170030180 & 7.5 & $4,50,000$ \\
\hline 5 & P. Akshay & 170030021 & 8.8 & $5,00,000$ \\
\hline 6 & A.Sasidhar & 170030172 & 6.5 & $1,50,000$ \\
\hline 7 & Rohithgada & 170031135 & 8.5 & $5,62,000$ \\
\hline 8 & k. Snehith & 170030651 & 9.8 & $7,00,000$ \\
\hline 9 & K. Neeraj & 170030643 & 9.5 & $6,20,000$ \\
\hline
\end{tabular}

Generally, We can expect that the students who have gained a good score/ percentage of marks in their semester, will be we placed in MNC's with a high-Annual Salary package, and the students, who have a low academic score in their academic subject will be placed in MNC's with low- Annual Salary Package.Table 5 depicts that the S.G.P.A of the semester and the Annual Salary Package that he/she gained during the Placements.Table 6 depicts the OPA score that he/she got generated by the Expert system and the AnnualSalary package of him/her.

Table 6: computed Performance of the students during Campus placement

\begin{tabular}{|c|c|c|c|c|}
\hline $\begin{array}{l}\text { S.N } \\
\text { O }\end{array}$ & Name & $\begin{array}{l}\text { Registrati } \\
\text { on } \\
\text { Number }\end{array}$ & $\begin{array}{c}\text { OPA score } \\
\text { Generated by } \\
\text { System } \\
\operatorname{Max}(10)\end{array}$ & $\begin{array}{c}\text { Annual- } \\
\text { Salary } \\
\text { Package } \\
\text { that he/she } \\
\text { Gained }\end{array}$ \\
\hline 1 & K.Sumanth & $\begin{array}{l}1700311 \\
61\end{array}$ & 6.5 & $6,00,000$ \\
\hline 2 & Rishi Ramana Sai & $\begin{array}{l}1700301 \\
18\end{array}$ & 7.6 & $6,40,000$ \\
\hline 3 & C. Akhil & $\begin{array}{l}1700305 \\
62\end{array}$ & 3.8 & $4,00,000$ \\
\hline 4 & ch Naga Saketh & $\begin{array}{l}1700301 \\
80\end{array}$ & 4.2 & $4,50,000$ \\
\hline 5 & P. Akshay & $\begin{array}{l}1700300 \\
21\end{array}$ & 4.4 & $5,00,000$ \\
\hline 6 & A.Sasidhar & $\begin{array}{l}1700301 \\
72\end{array}$ & 4.7 & $1,50,000$ \\
\hline 7 & Rohithgada & $\begin{array}{l}1700311 \\
35\end{array}$ & 5.6 & $5,62,000$ \\
\hline 8 & k. Snehith & $\begin{array}{l}1700306 \\
51\end{array}$ & 8.0 & $7,00,000$ \\
\hline 9 & K. Neeraj & $\begin{array}{l}1700306 \\
43\end{array}$ & 7.0 & $6,20,000$ \\
\hline
\end{tabular}

Based on the Results of students, two graphs were plotted. The Figure 2 is a plot drawn plotting S.G.P.A on Y-Axis and Annual salary package that he/she got while placed in MNC's on X-Axis. Figure 3 was plotted by taking the
Annual Salary package that he/she cracked in the Placements on X-Axis and the OPA score that he/she gained on Y-Axis.

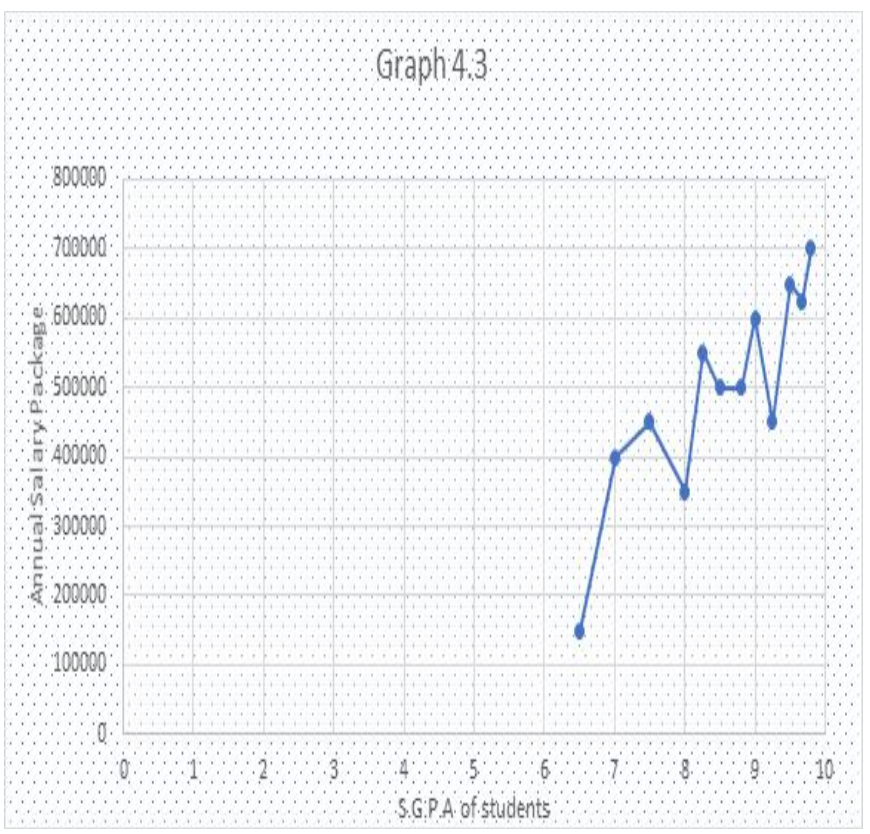

Figure 2: Performance of the students during placement in relation to SGPA

From Figure 2,we can observe that some students who had Scored less S.G.P.A in the Descriptive Exams have gained a good salary-Package than expected and there were another category of Students who have Excelled in S.G.P.A but gained low Annual Salary Package than expected from them.By this, we can understand that our Academic Results are not so accurate so at predicting the suitable Career Jobs for students.

\section{Graph 4.4}

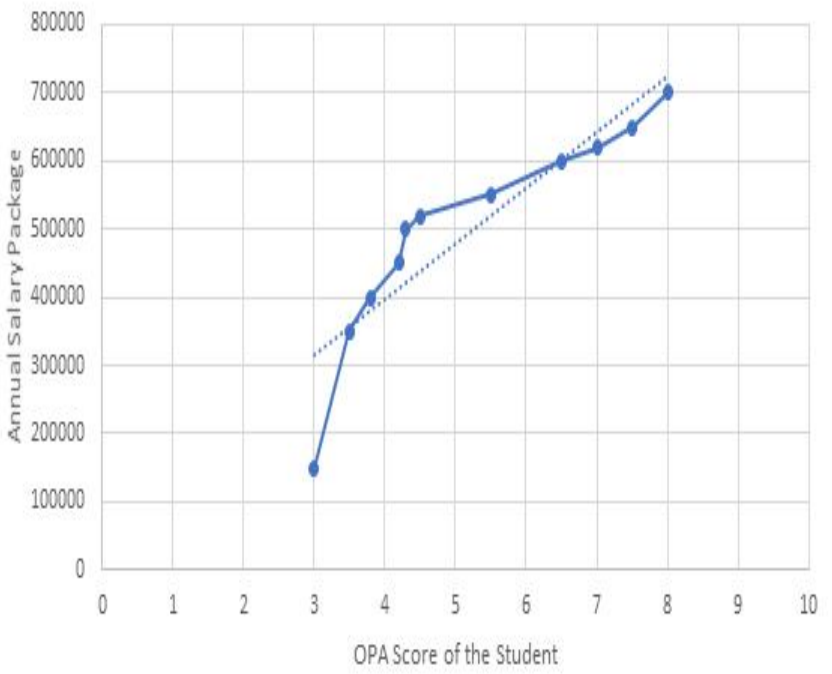

Figure 3 :Performance of the students during placement in relation to calculated score of the student by the expert system 
P. Vamsi et al., International Journal of Emerging Trends in Engineering Research, 8(5), May 2020, 2065 - 2073

OPA scores is computed using a 10 Point scale, converting the Qualitative score into a 10 Point scare and considering actual CGPA achieved by the students. From Figure 3, we can observe that students who are having good OPA score have successfully got placed in the MNC's with high Salary Package compared to the Students with low OPA score.This can show us a fact that the person with high OPA score are highly talented people and also possess qualities required for that Job in their Respective Domain The Students with low OPA score can be of two types, One who are highly talented but lacks the psychological factors required for their Job field .The Second type can be of those who are neither highly talented nor possessing the Psychological factors which mayhave endthem up with Low-Annual Salary Package

\section{CONCLUSION}

Assessment of Psychological factors is necessary for predicting suitable careers to the students. Assessment of Psychology of the student's trough game playing is the most appropriate approach. For doing a Job, student must possess certain level of Psychology.

The Kind of Psychology and the level at which the Psychological factors required for doing a JOB can be predetermined through an Expert engine and the same can be used for selecting suitable carriers to the students.

The kind of psychologicalfactors that can be determined vary from game to game. Some type of factors can be determined through more than one game and the level of the factors can vary drastically when determined using different game. There is a need to normalise the values and smoothen the same before the same can be used as the basis for predicting suitable careers to the students

Snakes and ladder game with built in rules can be used for computing the Psychology of the students considering the factors Knowledge, patience, perseverance, learning ability and the ability to memorise. The outcomes of this game can be combined with the outcomes of other

\section{ACKNOWLEDGMENT}

This exploration work is a part of a research project entitled "Development of an Expert System for career assessment based on cognitive model." funded by the Department of Science and Technology-Cognitive Science Research Initiative Scheme(CSRI) (Sanction Order No SR / CSRI/129/2014(G)) of the Government of India. Dr. V. Chandra Prakash is the project's Principal Investigator. K L (Deemed to be) University facilitates the infrastructure for the project

\section{REFERENCES}

1. Balasubramanyam, S., Padmaja Usharani, D., Harsha Vardhan Reddy, A., Swetha, D., Kumar, G. N. S., Anusha, K., \&Ahammad, S. H. (2018). Selecting a college academic branch-A design decision taking system for student career selection. International Journal of Engineering and Technology(UAE), 7(4.19 Special Issue 19), 323-

2. Beck, Aaron T., and Emily AP Haigh. "Advances in cognitive theory and therapy: the generic cognitive model." Annual review of clinical psychology 10 (2014): 1-24. https://doi.org/10.1146/annurev-clinpsy032813-153734.

3. Lent, Robert W., Ijeoma Ezeofor, M. Ashley Morrison, Lee T. Penn, and Glenn W. Ireland. "Applying the social cognitive model of career self-management to career exploration and decision-making." Journal of Vocational Behavior 93 (2016): 47-57. https://doi.org/10.1016/j.jvb.2015.12.007.

4. Steven K. Mtsweni and Johnson O. Dehinbo. "Development and Usability of a Web Application for Career Choice for High School Students." In Proceedings of the World Congress on Engineering and Computer Science, vol. 1. 2013.

5. Chathra Hendahewa, MaheshikaDissanayake" Artificial Intelligence approach to effective Career Guidance", Sri Lanka Association for Artificial Intelligence, 15th September 2006 - Colombo.

6. Manisha L Waghmode, P PJamsandekar, Expert system for Career Selection: Theoretical Framework, Volume 4, Issue 8,August 2016 International Journal of Computer Science, ISSN: 2321-5992.

7. El Haji, Essaid, AbdellahAzmani, and Mohamed El Harzli. "Multi-expert system design for educational and career guidance: an approach based on a multi-agent system and ontology." International Journal of Computer Science Issues (IJCSI) 11, no. 5 (2014): 46. https://doi.org/10.1109/ICMCS.2014.6911256

8. Saraswathi, S., M. Hemanth Kumar Reddy, S. Udaya Kumar, M. Suraj, and SkKhajaShafi. "Design of an online expert system for career guidance." The International Journal of Research in Engineering and Technology 3 (2014).

9. Ezenkwu, C. P., E. H. Johnson, and O. B. Jerome. "Automated Career Guidance Expert System Using Case-Based Reasoning."; Computing, Information Systems, Development Informatics \& Allied Research Journal.

10. Athanasios Drigas, Stelios Kouremenos, Spyros Vrettos, John Vrettaros, and Dimitris Kouremenos. "An Expert sys-tem for job matching of the unemployed." Expert Systems with Applications26, no. 2 (2004): 217224. https://doi.org/10.1016/S0957-4174(03)00136-2.

11. Essaid El Haji, AbdellahAzmani, and Mohamed El Harzli. "Expert system design for educational and vocational guidance, using a multi-agent system." In Multimedia Computing and Systems (ICMCS), 2014 International Conference on, pp. 1018-1024. IEEE, 2014.

https://doi.org/10.1109/ICMCS.2014.6911256

12. Sripath Roy, K., Roopkanth, K., UdayTeja, V., Bhavana, V., \& Priyanka, J. (2018). Student career prediction using advanced machine learning techniques. 
P. Vamsi et al., International Journal of Emerging Trends in Engineering Research, 8(5), May 2020, 2065 - 2073

International Journal of Engineering and Technology (UAE), 7(2), 26-29.

13. Chandra Prakash, V., Sastry, J. K. R., (2018). A critical study on applicability of sokoban game for building cognitive models of a student for career assessment. International Journal of Engineering \& Technology, 7 (1.1) (2018) 260-264. https://doi.org/10.14419/ijet.v7i1.1.9482

14. V. Chandra Prakash, J. K. R.Sastry, K.B.Anusha, A.B.Spandana, N. Dhatrija, V.Nikhi, Applicability of Sudoku game for building the cognitive model of a student for career assessment -an analytical study, International Journal of Engineering \& Technology, 2018, 7 (1.1), pp 246-251

15. Dr.SasiBhanuJ, Dr.Baswaraj, Mrs. Sunitha Devi Bigul, Dr.JKRSastry, Career Prediction through Cognitive Models using Sudoku Game - The Assessment of Applicability, International Journal of Engineering Trends and Engineering Research, 2019, Vol. 7 Issue 11, pp. 477-480

16. V. Chandra Prakash, J. K. R. Sastry, K. Anusha, P. Ashok Kumar, N. Venkatesh, G. Ravi Teja, Expert system for building cognitive model of a student using 8-puzzle game and for career Assessment, International Journal of Engineering \& Technology, 2018, 7 (2.27) pp, 113-117 https://doi.org/10.14419/ijet.v7i2.27.12014

17. Dr. J. SasiBhanu, Dr.JKRSastry, B. Sunitha Devi, Dr. V Chandra Prakash, Career Guidance through TIC-TACTOE Game, International Journal of Engineering Trends and Engineering Research, 2019, Vol. 7 Issue 6, pp. 2531 https://doi.org/10.30534/ijeter/2019/01762019

18. J. SasiBhanu, JKRSastry, V Chandra Prakash, Assessing the intelligence of a student through tic-tac-toe game for career guidance, International Journal of Electrical and
Computer Engineering (IJECE), 2019, Vol. 9, No. 6,, pp. 5545 5551

19. Dr. V. Chandra Prakash, Dr. J. K. R. Sastry ,V. Kantharao, V. Sriharshini, G. Sriram, C.H.V.S. Ganesh, AN EXPERT SYSTEM TO ASSESS MEMORY POWER OF A STUDENT FOR SELECTION OF A SUITABLE CAREER, Journal of Advanced Research in Dynamical and Control Systems, 2017, Vol. 9. Sp6, pp. 309-321

20. V. Kantha Rao, Dr. V. Chandra Prakash, A.Jyothsana ${ }^{3}$, T.Sainadh, P.Harshitha ${ }^{5}$ Assessing Psychological Factors of a Student Through Concentration Game for Career Selection, International Journal of Engineering \& Technology,2018, 7 (2.32), pp. 443-445 https://doi.org/10.14419/ijet.v7i2.32.15736

21. V. Chandra Prakash, J.K.R. Sastry, B. Tirapathi Reddy' J. S. Ravi Teja, A. Bala Venkatesh, M. S. K. Vamsi Varma" An Expert System for building a Cognitive and Career Prediction model based on N-Coin Puzzle Game, International Journal of Engineering Trends and Engineering Research, 2019, Vol. 7 Issue 11, pp. 410416

22. V. Chandra Prakash, V. Kantharao, JKRSastry, V. Bala Chandrika, Expert system for building Cognitive model of a student using Crypt Arithmetic game and for Career Assessment, International Journal of Recent Technology and Engineering (IJRTE), 2019, Volume-7, Issue-6S, pp. 684-689

23. V. Chandra Prakash, J.K.RSastry' G.Reeshmika, M.Pavani, P.Chikitha Sree, J.S Ravi Tej, Development of a Comprehensive and Integrated Expert System for Career Assessment based on Cognitive models, International Journal of Engineering Trends and Engineering Research, 2019, Vol. 7 Issue 11, pp. 617627

https://doi.org/10.30534/ijeter/2019/347112019 
P. Vamsi et al., International Journal of Emerging Trends in Engineering Research, 8(5), May 2020, 2065 - 2073

Table 4: Job requirements in terms of Psychological and Academic achievement in the concerned subjects

\begin{tabular}{|l|l|l|l|l|l|l|}
\hline \multicolumn{1}{|c|}{ Job Role } & $\begin{array}{c}\text { Patience } \\
\text { level }\end{array}$ & $\begin{array}{c}\text { Perseverance } \\
\text { level }\end{array}$ & $\begin{array}{c}\text { Learning } \\
\text { Ability }\end{array}$ & \multicolumn{1}{|c|}{ Knowledge } & $\begin{array}{c}\text { Data } \\
\text { structures\& } \\
\text { Algorithms }\end{array}$ & \multicolumn{1}{|c|}{ Software Engineering } \\
\hline $\begin{array}{l}\text { Software } \\
\text { Developer }\end{array}$ & Medium & Medium & High & Distinction & Distinction & Distinction \\
\hline $\begin{array}{l}\text { Maintenance } \\
\text { Engineer }\end{array}$ & High & Medium & Medium & $\begin{array}{l}\text { First Class(or) } \\
\text { above }\end{array}$ & $\begin{array}{l}\text { First } \\
\text { Class(or) } \\
\text { above }\end{array}$ & First Class(or) above \\
\hline Tester & High & High & High & Distinction & $\begin{array}{l}\text { First } \\
\text { Class(or) } \\
\text { above }\end{array}$ & First Class(or) above \\
\hline $\begin{array}{l}\text { Sales } \\
\text { Executive }\end{array}$ & High & High & Medium & $\begin{array}{l}\text { Second } \\
\text { Class(or) above }\end{array}$ & $\begin{array}{l}\text { Second } \\
\text { Class(or) } \\
\text { above }\end{array}$ & Second Class(or) above \\
\hline
\end{tabular}

